\title{
Yeast-based production and in situ purification of acetaldehyde
}

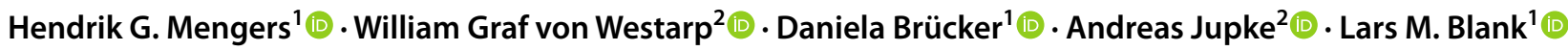

Received: 15 September 2021 / Accepted: 19 January 2022 / Published online: 8 February 2022

(c) The Author(s) 2022

\begin{abstract}
Acetaldehyde is a platform chemical with a production volume of more than $1 \mathrm{Mt} / \mathrm{a}$, but is chiefly synthesized from petrochemical feedstocks. We propose the fermentative conversion of glucose towards acetaldehyde via genetically modified $S$. cerevisiae. This allows for ethanol-free bioactaldehyde production. Exploiting the high volatility of the product, in situ gas stripping in an aerated reactor is inevitable and crucial due to the respiratory toxicity effects of the acetaldehyde overproduction. We devise a lab-scale setup for the recovery of the product from the off-gas. Water was chosen as a suitable solvent and the Henry coefficient of acetaldehyde in water was validated experimentally. Based on an experimentally verified capture efficiency of $75 \%$, an acetaldehyde production rate of over $100 \mathrm{mg} / \mathrm{g} / \mathrm{h}$ was reached in $200 \mathrm{~mL}$ lab-scale fermentations.
\end{abstract}

Keywords Acetaldehyde $\cdot$ S. cerevisiae $\cdot$ In situ gas stripping $\cdot$ Absorption $\cdot$ Redox balance

\section{Introduction}

The chemical industry needs to go green(er). This is necessary to reach the goal of climate neutrality within the next three decades as demanded by the European Union [1]. The chemical industry uses over $650 \mathrm{Mt} / \mathrm{a}$ of petrochemical feedstocks and, on top of that, over $510 \mathrm{Mt} / \mathrm{a}$ of hydrocarbons for process energy [2]. Besides costly Power-to-X approaches, biotechnological production of platform chemicals from renewable carbon sources (i.e. biomass, $\mathrm{CO}_{2}$, and waste) can reduce and at one point eliminate the use of fossil resources.

In industrial biotechnology, Saccharomyces cerevisiae is one of the most frequently used hosts, with the bioethanol sector as the prime example reaching $76.5 \mathrm{Mt} / \mathrm{a}$ in 2015 [3]. With nowadays easy genetic modifications, the feedstocks can range from glucose over lignocellulose biomass, $\mathrm{H}_{2} / \mathrm{CO}_{2}$ up to plastic monomers and crude oil $[4,5]$. The range of products is almost limitless, ranging from alkenes, alcohols, organic acids over pyridines up to peptides, enzymes, and pharmaceutical proteins. However, the efficient purification

Lars M. Blank

lars.blank@rwth-aachen.de

1 Institute of Applied Microbiology - iAMB, Aachener Biologie und Biotechnologie - ABBt, RWTH Aachen University, Aachen, Germany

2 Fluid Process Engineering (AVT.FVT), RWTH Aachen University, Aachen, Germany of these products from the aqueous medium remains challenging and is the primary factor in manufacturing costs for the overwhelming majority of products [6].

Acetaldehyde is a large-volume commodity chemical, as it is a possible precursor for acetic acid, pyridines, as well as 1,3-butylene glycol. Further, it is used as a fine chemical in food and flavour industries. The global market consumption of acetaldehyde exceeds $1 \mathrm{Mt} / \mathrm{a}$ and is expected to grow in the next decade $[7,8]$. The primary production route is through the Wacker-Hoechst process, the partial oxidation of ethylene, which in turn is mostly obtained from steamcracked fossil resources [9]. A major field of research is the catalyst-driven dehydrogenation of bioethanol to acetaldehyde which enables acetaldehyde production based on renewable resources, hence bioacetaldehyde production [10-12]. For example, the Pörner Group offers this as a pre-planned industrial facility [13]. Although this process is already used in industrial scale, it can be enhanced in terms of efficiency, as acetaldehyde is an intermediate in bioethanol production, and $15 \%$ of the bioethanol production cost emerge in the downstream processing [14] A direct bioacetaldehyde production would circumvent the need for two purification steps.

Acetaldehyde as the precursor of ethanol in S. cerevisiae was discovered in the 1910s, among others by the work of Neuberg, although he targeted glycerol production. He used calcium sulfite to chemically bind the nascent acetaldehyde in yeast ferments in order to accumulate glycerol [15]. 
The next step for the compound's overproduction was the establishment of Zymomonas mobilis as a production host by Wecker et al. in the 1980s, using the natively upregulated NADH oxidase activity in aerobic environments and thus limiting the reduction of acetaldehyde to ethanol. The volatile acetaldehyde is stripped from the fermenter and is bound by reacting with an aqueous sodium bisulfite solution in a subsequent trap. In this first targeted fermentation towards acetaldehyde, yields of $40 \%$ and a capture efficiency of $80 \%$ were achieved $[16,17]$. Tanaka et al. optimized this method and further explored the combination of water traps and reactive precipitation via a 3-methyl-2-benzothiazolinone hydrazone hydrochloride solution in consecutive traps. This method gave a 94\% overall recovery of acetaldehyde [18]. In Kalnenieks et al., the expression levels of the NADH oxidase, as well as the alcohol dehydrogenase were changed through genetic modifications. This resulted in very high yields, but this study circumvented the recovery of acetaldehyde by measuring the concentration in the off-gas [19]. Other works focus on establishing new host organisms by introducing the $Z$. mobilis pyruvate decarboxylase gene into other microorganisms [20,21].

All these approaches rely on limited acetaldehyde to ethanol conversion but cannot prevent ethanol production entirely. We propose for the first time bioacetaldehyde production without the co-production of ethanol. For that, we chose $S$. cerevisiae as the main host of the closely related ethanol production. Further, we strive to bypass the need for reactive compounds to bind acetaldehyde, as Wecker et al. and Tanaka et al. did. Hence, we follow a different approach by using water traps only and demonstrating the capture efficiency of traps in a lab-scale proof of concept. Rightfully, industrial volatile organic compound (VOC) emissions are heavily restricted [22]. In order to comply with these restrictions, the proposed system with water traps is not sufficient. To release the exhaust gas with the desired purity and under usage of reduced solvent demand, an absorption column in counter-current mode would be required [23]. The usage of an absorber column was already suggested for a large-scale process for the production of acetaldehyde from ethanol. Here, the acetaldehyde is produced in the gas phase and then absorbed to water. Consecutively the acetaldehyde is separated from water in a distillation step [24]. However, such a system is not applicable in lab-scale, which is why it is not further investigated in this study.

To overproduce acetaldehyde without adding high amounts of reactive chemicals in yeasts, as Neuberg et al. did, the reaction towards ethanol needs to be eliminated [15]. This can be done by deleting all alcohol dehydrogenases (ADHs) and enzymes with ADH activities. This deprives the yeast of its ability to grow under anaerobic conditions, as the redox cofactor NADH cannot be regenerated through the formation of ethanol. Therefrom results the need to manage the carbon flux at the pyruvate branch (see Fig. 1). In aerobic environments, normally, all glycolysis products flow into the tricarboxylic acid cycle (TCA) and the electrons bound in NADH subsequently towards the respiratory chain. S. cerevisiae belongs to the Crabtree-positive yeasts. After exceeding a glucose threshold of only $0.1 \mathrm{~g} / \mathrm{L}$ depending on the strain, respiration is down-regulated, and the excess pyruvate is

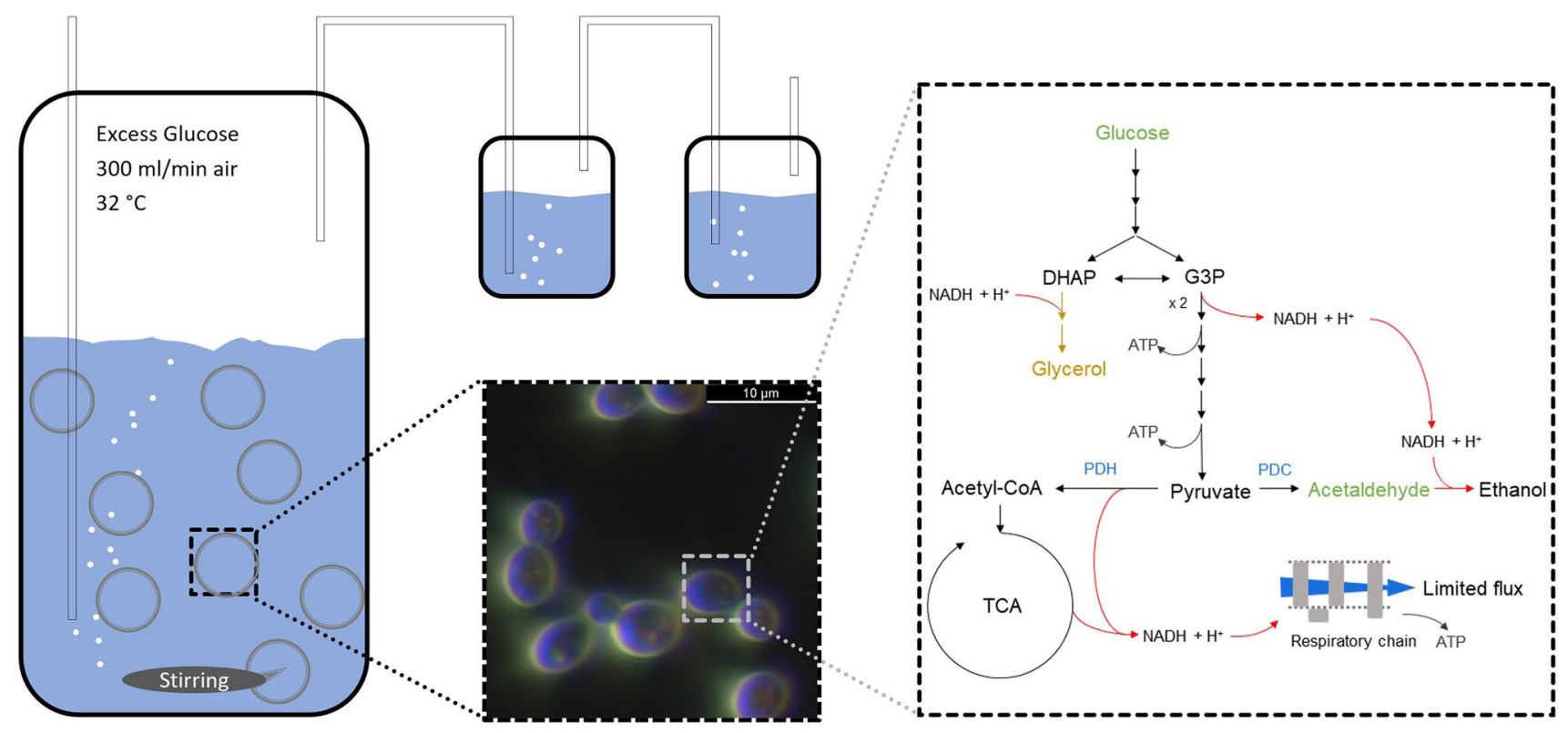

Fig. 1 Production concept and network graph of acetaldehyde and generated ATP and NADH during glycolysis, the TCA, and ethanol production. In blue are the enzymes of the pyruvate branching: the pyruvate dehydrogenase (PDH, left) and the pyruvate decarboxylase (PDC, right) 
transferred to the ethanol branch via the conversion to acetaldehyde [25, 26]. This work develops a novel fermentative production strategy of acetaldehyde based on renewable resources with genetically modified $S$. cerevisiae. The in situ stripping of acetaldehyde is not only inevitable in aerated fermenters but also favourable to reduce the toxic effect of acetaldehyde [27]. Obviously, stirring, aeration rates, and the fermentation temperature have a significant impact on the evaporation rates, but their optimization is out of scope for this work. In a consecutive step, we implement and investigate suitable separation techniques to capture the acetaldehyde from the gas stream to quantify the acetaldehyde.

\section{Materials and methods}

\section{Used strain}

The strain used is based on the $S$. cerevisiae CEN.PK11317 a including knockouts for all seven alcohol dehydrogenases and in genes for two multifunctional alcohol dehydrogenases, namely $S F A 1$ and GRE2. Thus the genotype is MATo; ura3-52; leu2-3_112; TRP1; HIS3; MAL2-8C; SUC2, $\Delta a d h 1, \Delta a d h 2, \Delta a d h 3, \Delta a d h 4, \Delta a d h 5, \Delta a d h 6, \Delta s f a 1, \Delta g r e 2$ [26]. This is a haploid laboratory strain chosen for the ease of using the leucine deficiency for CRISPR/Cas9-assisted genetic engineering.

\section{Yeast cultivation and media}

Precultures were grown in liquid yeast extract peptone (YEP, for a more detailed composition see S1.1) medium at $30{ }^{\circ} \mathrm{C}$ in shaking flasks. Main cultures were grown in Verduyn minimal medium or optimized Verduyn minimal medium $\left(\mathrm{V}^{+}\right.$, for a more detailed composition see S1.1) [28]. For the fermentations, a double-walled glass reactor with a working volume of up to $250 \mathrm{~mL}$ was used but only filled with $200 \mathrm{~mL}$ medium. The reactor was heated with a thermostat to 30 or $32{ }^{\circ} \mathrm{C}$ and stirred with the maximum possible speed of around $800 \mathrm{rpm}$ with a triangular, $3.7 \mathrm{~cm}$-long stirring bar. Compressed, humidified air was introduced via a needle with $0.8 \mathrm{~mm}$ diameter at $1.5 \mathrm{vvm}$ (i.e. $300 \mathrm{~mL} / \mathrm{min}$ ) (for a more detailed setup see S1.2, Fig. S1.1, and Fig. 1). Higher aeration rates facilitate acetaldehyde evaporation but at the same time hinder acetaldehyde capture, therefore $1.5 \mathrm{vvm}$ was chosen as upper limit of standard fermentation procedure [29].

The optical density at $600 \mathrm{~nm}$ was determined with a Ultrospec 10 (Amersham Biosciences, Little Chalfont, UK) photometer. Cell dry weight (CDW) was determined through a calibration curve following the growth of the strain in minimal media, yielding a correlation of 0.398 on OD/(g/L) CDW (for more detailed results see S2.1).

\section{GC and HPLC analytics}

For GC-FID analytics, all samples were diluted in a ratio of 1:20 with acetonitrile (ACN) and directly stored in a screwcap GC-vial at $4{ }^{\circ} \mathrm{C}$. Samples that contained biomass were filtered through Berrytec CA $0.22 \mu \mathrm{m}$ disposable syringe filters before dilution. Samples with high glucose content, e.g. uninoculated medium, tend to precipitate upon dilution; those samples were centrifuged for $2 \mathrm{~min}$ at $10,000 \times \mathrm{g}$ and decanted before storage in GC vials. A Trace GC Ultra GCFID (Thermo Scientific, Waltham, MA, USA) was used with a $30 \mathrm{~m}$ Zebron ZB-WAX column with an inner diameter of $0.25 \mathrm{~mm}$. The optimized sequence parameters are the following: a sample volume of $0.1 \mathrm{~mL}$, a flow rate of $1 \mathrm{~mL} / \mathrm{min}$ with helium as carrier gas and a split ratio of 1:10. The temperature program was as follows: $50^{\circ} \mathrm{C}$ for $8 \mathrm{~min}$, increased by $27.5^{\circ} \mathrm{C} / \mathrm{min}$ to $160^{\circ} \mathrm{C}$, and kept constant for $3 \mathrm{~min}$. The inlet was kept at $250^{\circ} \mathrm{C}$.

For HPLC analytics, all samples were filtered through Berrytec CA $0.22 \mu \mathrm{m}$ disposable syringe filters before analysis. A DIONEX UltiMate 3000 HPLC System (Thermo Scientific, Waltham, MA, USA) with a Metab-AAC column $(300 \times 7.8 \mathrm{~mm}$ column, ISERA, Düren, Germany) was used. Elution was performed with $5 \mathrm{mM} \mathrm{H}_{2} \mathrm{SO}_{4}$ at a flow rate of $0.4 \mathrm{~mL} / \mathrm{min}$ and a temperature of $40{ }^{\circ} \mathrm{C}$. For detection, a SHODEX RI-101 detector (Showa Denko Europe GmbH, München, Germany) and a DIONEX UltiMate 3000 Variable Wavelength Detector set to $210 \mathrm{~nm}$ were used.

\section{Determination of Henry coefficients}

Property prediction for acetaldehyde and multiple solvents was performed by screening charges calculated by the conductor-like screening model (COSMO) using COSMOthermX19. COSMO for real solvation (COSMO-RS) was applied to gain activity coefficients by using the COSMOtherm internal BP-TZVPD-FINE parametrization, which refers to the Becke-Perdew functional and the triple zeta valence plus polarization function [30]. Based on the activity coefficients, the Henry coefficient of acetaldehyde in a solvent was determined at $25^{\circ} \mathrm{C}$.

For the experimental validation of the Henry coefficient, the dynamic equilibrium was determined by applying the gas stripping method as described in previous studies [31, 32]. Acetaldehyde was inserted into a Schott flask with three welldefined feeding rates of $0.8-2.1 \mathrm{mmol} / \mathrm{L} / \mathrm{h}$. The flask is flooded by an airstream with a flow rate of $300 \mathrm{~mL} / \mathrm{min}$, leading to a partial pressure of acetaldehyde in a range between 0.0011 and 0.0031 bar. The airstream was introduced to a water trap containing $250 \mathrm{~mL}$ of liquid. Samples with a volume of $0.5 \mathrm{~mL}$ were taken from the trap for $24 \mathrm{~h}$ as described above and were analysed in the GC (for a more detailed setup see S1.2, for more detailed results S2.5). Based on the defined amount of 
acetaldehyde in the entering gas stream and the measured concentration of acetaldehyde in the first trap when in a stationary state, equilibrium was assumed, and the Henry coefficient $H_{i}$ was determined as follows:

$x_{i} \cdot H_{i}=p_{i}$

$\frac{p_{i}}{p}=y_{i}=\frac{n_{i}}{n_{\mathrm{gas}}}$

$x_{i}=\frac{c_{i} \cdot V_{\text {liq }}}{n_{\text {liq }}}$,

where the partial pressure $p_{i}$ was calculated as the quotient of the molar amount of acetaldehyde $n_{i}$ and the total molar amount of the gas phase $n_{\text {gas }}$ by applying the ideal gas model. The molar fraction $x_{i}$ in the liquid phase was calculated via the measured concentration $c_{i}$, the molar amount of acetaldehyde $n_{\text {liq }}$ and the liquid volume $V_{\text {liq }}$ in the water trap.

\section{Results and discussion}

With an engineered S. cerevisiae in hand that did not produce any ethanol, a first proof of concept, a fermentation at a $200 \mathrm{~mL}$ scale was conducted. The aeration was set to $1.5 \mathrm{vvm}$. With the expected stripping of acetaldehyde, a concept for the recovery from the gas stream was needed. Given the boiling point of acetaldehyde at $20.8{ }^{\circ} \mathrm{C}$, the easiest solution seemed to be the condensation from the off-gas. A glass condenser at $0{ }^{\circ} \mathrm{C}$ with an inner surface of roughly $170 \mathrm{~mm}^{2}$ was used.

The strain grew in the first $24 \mathrm{~h}$ of the fermentation to a density of approximately $2.8 \mathrm{~g} / \mathrm{L} \mathrm{CDW}$. Samples taken from the reactor, as well as from the condensed off-gas, were analysed in the GC-FID and did not include any ethanol, confirming the knockout of the alcohol dehydrogenases to be successful and no alternative enzyme activity existing. At the end of the fermentation, a total of $22 \mathrm{~mL}$ liquid was found in the cold trap with an acetaldehyde concentration of $8.8 \mathrm{mmol} / \mathrm{L}$. No traces of other volatile components were found in the GC measurement. The estimated yield on glucose was in the order of $0.2 \%$ (for more detailed results see S2.2).

\section{Efficiency test for condensers}

Due to the distinct smell of acetaldehyde in the off-gas, we hypothesised that the product was not condensed entirely. Thus, we devised an efficiency test. The capture efficiency can be determined by injecting a known amount of acetaldehyde into the system and measuring the acetaldehyde in the condensate and the reactor. Since the fermentation produces acetaldehyde over time, rather than injecting all acetaldehyde at once, a syringe pump was used to inject a constant feed over $16 \mathrm{~h}\left(4.7 \mathrm{~g}\right.$ in $50 \mathrm{~mL} \mathrm{H}_{2} \mathrm{O}$ with $\left.3.1 \mathrm{~mL} / \mathrm{h}\right)$. The capture efficiency is calculated as acetaldehyde found in the condensate divided by acetaldehyde injected minus the amount left in the reactor. From the injected $107.3 \mathrm{mmol}$, only $0.76 \mathrm{mmol}$ were found in a total of $3.5 \mathrm{~mL}$ of condensate. The experiment showed an efficiency of approximately $0.7 \%$, but multiple runs showed clearly diverging results (for more detailed results see $\mathrm{S} 2.3$ ).

The low amounts captured via the condenser can be explained by the high dilution of acetaldehyde in the gas stream. Based on the approximated capture efficiency, the partial pressure of acetaldehyde can be estimated to be 0.0005 bar by assuming ideal gas conditions. For the condensation of a component, the partial pressure needs to exceed its saturation pressure at the given temperature. When comparing the partial pressure of 0.0005 bar with the saturation pressure of $0.351 \mathrm{bar}$ at $0{ }^{\circ} \mathrm{C}$ calculated based on Antoine parameters from literature [33], it is evident that the partial pressure of acetaldehyde is lower by three orders of magnitude than the saturation pressure. Despite these calculations, little amounts of acetaldehyde were found in the condenser trap. This can be attributed to the idealised estimation of the ideal gas model or to the high solubility of the acetaldehyde in the condensed water. Hence, these results demanded a new method for product recovery.

\section{Absorption}

For the purification of polluted gas streams, absorption is a standard procedure. The absorption of acetaldehyde either as a toxic component from room air or as a fermentation product has been investigated in these studies [16, 34]. However, the acetaldehyde was bound to amino acids or sodium bisulphite, respectively, via reactive absorption and was not processed further. This approach leads to an additional process step in purification, as the reactively bound acetaldehyde needs to be released. The release of the bound acetaldehyde can be achieved by the addition of a base, which in turn produces waste salts. In this study, we focus on developing an absorption procedure, which enables us to capture the acetaldehyde from a diluted gas stream and obtain it as a product solution. Therefore, we absorbed the produced acetaldehyde into an aqueous medium and could, in theory, increase the concentration of the acetaldehyde in the binary mixture via consecutive distillation. With this approach, we are able to collect acetaldehyde to quantify the produced amount in the fermentation.

Due to its simple handling and non-toxicity, water is an absorption medium of favour in this proof of concept study. To assess the gas solubility in equilibrium of acetaldehyde in water and to compare it to traditional organic solvents, the Henry coefficient was determined using the conductor-like 
screening model for real solvations (COSMO-RS) [35]. A small Henry coefficient indicates that the equilibrium for a component is strongly on the side of the liquid phase. Another criterion for the absorbent choice was the vapour pressure of the solvents [23].

Water was compared with eight organic solvents as an absorbent. Its relatively low predicted Henry coefficient (3.098 bar) and a sufficiently low vapour pressure of 0.0317 bar make it a reasonable choice of absorption medium (for more details see S2.4). Another advantage of water compared to the proposed organic solvents is that the water content in the off-gas has not to be considered as an additional component in further processing. The experimental determination of the Henry coefficient is based on the known entering acetaldehyde stream and the measured acetaldehyde concentration in one water trap. When the concentration in the water does not change over time, the two phases are assumed to be in equilibrium (for more details see S1.2 and S2.5). In Fig. 2, the molar fraction of acetaldehyde in water (horizontal axis) was correlated with the partial pressure of acetaldehyde in the gas phase (vertical axis). Three measurement points are depicted, representing partial pressures of acetaldehyde between 0.0011 bar and 0.0032 bar. A regression line was elongated through the measurement points and the origin. The slope through the measurement points and the origin represents the corresponding Henry coefficient. Hence, the experimentally derived Henry coefficient was determined to be 2.592 bar. The Henry coefficient derived from COSMO-RS is larger (3.098 bar) than the experimentally determined one, representing a lower affinity of acetaldehyde towards the absorbent. The larger Henry coefficient was chosen to estimate the minimal amount of solvent for the design of the absorptive separation sequence in order to conduct the estimation to the safe site.

The necessary amount of water for capture efficiencies of more than 70\% in batch mode increases rapidly (see S2.9). This is due to a doubled demand of water to halve the acetaldehyde content remaining in the gas phase. Hence, a capture efficiency of $70 \%$ was targeted and the minimum amount of water needed to achieve this goal was calculated. In order to dissolve $70 \%$ of the inserted acetaldehyde from a gas stream of $300 \mathrm{~mL} / \mathrm{min}$ over $16 \mathrm{~h}$ in the absorbent, approximately $1570 \mathrm{~mL}$ of water are necessary, assuming that the system consisting of water and gas phase is in equilibrium. Hence, a setup consisting of multiple consecutive water traps with a total water volume of $2000 \mathrm{~mL}$ was realized for the following experiments. The quantity of water traps was chosen to measure acetaldehyde accumulation over time and to obtain information about the equilibrium by analysis of the individual traps.

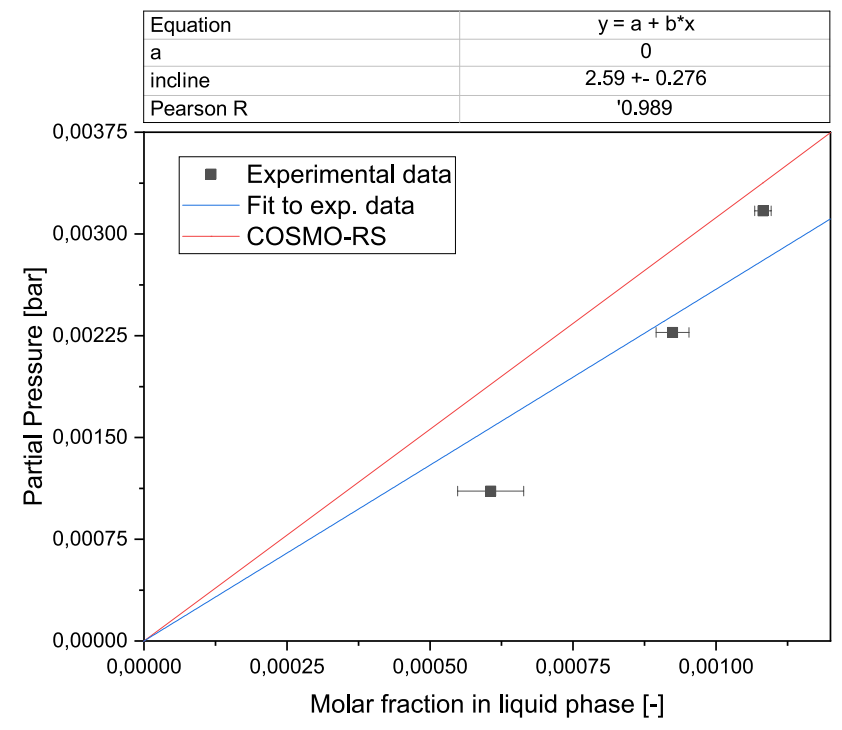

Fig. 2 Correlation of molar fraction of acetaldehyde in the liquid phase and the partial pressure of acetaldehyde in the gas phase in bar at $25{ }^{\circ} \mathrm{C}$. The slope of the black line refers to the predicted Henry coefficient using COSMO-RS calculations (3.098 bar). The experimentally derived measurement points are depicted as black squares. A linear regression line was calculated. The slope of the linear regression line represents the experimentally derived Henry coefficient which was determined to 2.592 bar. The experimentally determined Henry coefficient is in the same order of magnitude as the calculation based on COSMO-RS

\section{Efficiency tests in the water traps}

Instead of the condenser with a subsequent flask for acetaldehyde recovery, four water traps with $250 \mathrm{~mL}$ each and a fifth with $1000 \mathrm{~mL}$ were implemented. To enlarge the specific surface area of the air bubbles and thus enhance mass transfer, a sparger consisting of a tube with $0.8 \mathrm{~mm}$ holes was used in every trap. For a stable 1.5 vvm airflow despite the heightened resistance, the pressure was increased up to 1.4 bar. Although pressure influences the Henry coefficient in theory, small pressure changes below $10 \mathrm{bar}$ are neglectable [36]. To enhance the evaporation of acetaldehyde from the reactor, the fermentation temperature was increased to $32{ }^{\circ} \mathrm{C}$, the upper optimum limit for $S$. cerevisiae [37]. Since water was chosen as solvent, there was no need to remove moisture from the off-gas (for a more detailed setup see $\mathrm{S} 1.2$, for more detailed results see S2.6).

Analogous to the condenser tests, acetaldehyde was fed to the aerated reactor and the capture efficiency was calculated based on consecutive GC analysis. In total, this system is able to capture $75 \pm 3 \%$, along all tested loadings of acetaldehyde between 20 and $120 \mathrm{mmol}$ (see Fig. 3). With the determined constant capture efficiency of this setup, the amount of produced acetaldehyde in the following fermentations can be quantified. 


\section{Fermentative production of bioacetaldehyde in lab-scale}

The same strain was used again, but this time in an optimized medium with more trace elements, vitamins, and supplements $\left(\mathrm{V}^{+}\right.$, for more details see $\left.\mathrm{S} 1.1\right)$. A fed-batch operation mode was implemented with an initial glucose

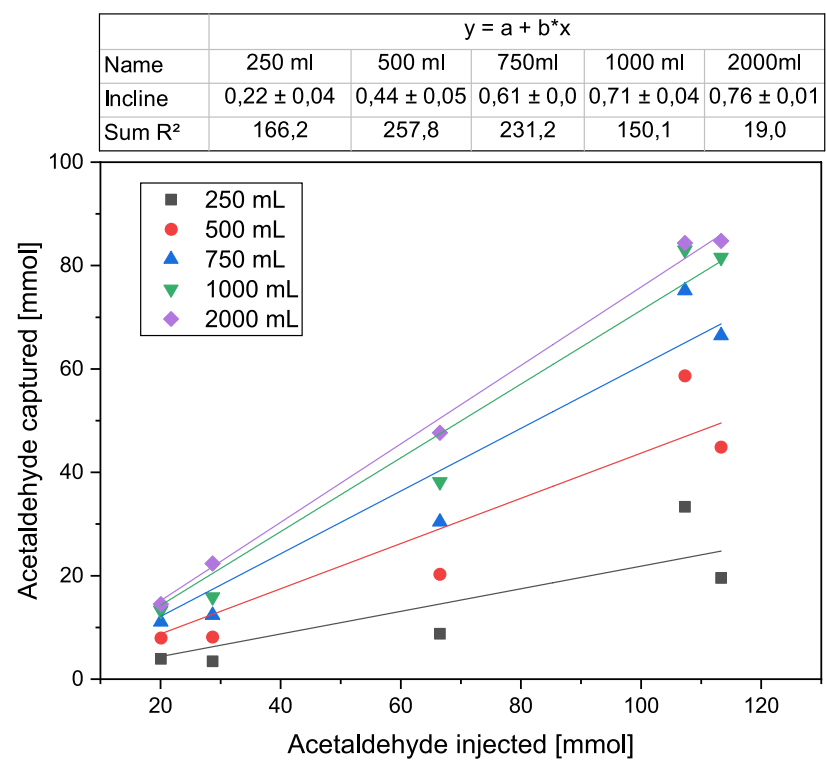

Fig. 3 Acetaldehyde trapping efficiency tests. Cumulative capture efficiency of the water trap system consisting of five consecutive traps for different amounts of acetaldehyde fed after $24 \mathrm{~h}$. The capture efficiency is calculated as acetaldehyde found divided by acetaldehyde injected minus the amount left in the reactor

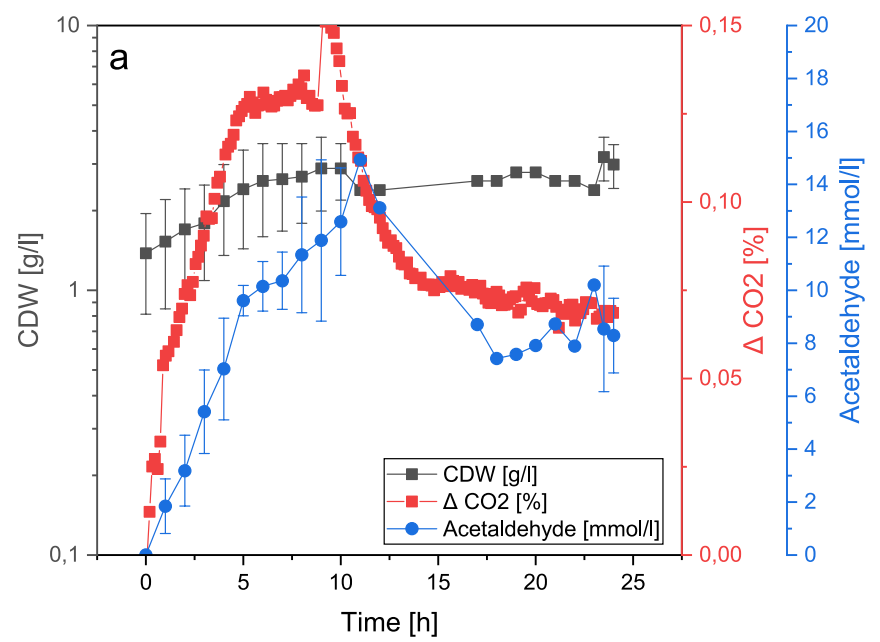

Fig. 4 Fermentations with acetaldehyde capture. a CDW, $\mathrm{CO}_{2}$ signal (difference between the carbon dioxide concentrations in $\mathrm{v} / \mathrm{v} \%$ of inflow air and off-gas), and reactor supernatant acetaldehyde concentration as a mean with standard deviation $(n=3$ for $\mathrm{h}$ $0-3,24 ; n=2$ for $4-10,23$ and $n=1$ for $12-23$, for $\mathrm{CO}_{2} n=3$ for $\mathrm{h}$ $0-9, n=2$ for 9-24). b Total amount of glycerol and acetaldehyde concentration of $20 \mathrm{~g} / \mathrm{L}$ at inoculation and a steady feed. The feed started after $3 \mathrm{~h}$ with $5 \mathrm{~g} / \mathrm{L} / \mathrm{h}$ for $16 \mathrm{~h}$ to ensure excess glucose conditions. To gain deeper insights into the cellular metabolism, two BlueSens $\mathrm{BCP}-\mathrm{CO}_{2}$ sensors were implemented; one before the reactor and one after the first four water traps. The $\mathrm{CO}_{2}$ signal is the difference between the carbon dioxide concentrations in $\mathrm{v} / \mathrm{v} \%$ of inflow air and off-gas.

Figure $4 \mathrm{a}$ shows biomass, $\mathrm{CO}_{2}$ signal, and acetaldehyde concentration in detail for one fermentation (for more detailed results see S2.7). The cells grew with a short lag phase and reached a stationary phase within the first $10 \mathrm{~h}$ of the fermentation. The $\mathrm{CO}_{2}$ signal increases after inoculation with the biomass, but declines after the cell reach the stationary phase. An increase in biomass beyond the peak cellular activity is most likely due to the usage of intracellular storage molecules.

But why is a steady-state reached at such low biomass concentrations? In all three fermentations, acetaldehyde accumulates in the reactor at concentrations of up to 10 to $15 \mathrm{mmol} / \mathrm{L}$. A non-changing concentration of acetaldehyde in the reactor means an equilibrium of production rate and gas stripping. This concentration is described to inhibit glucose-dependent respiration in S. cerevisiae [38]. This fits our data, as reaching this level directly correlates with the peak of the $\mathrm{CO}_{2}$ signal. The mechanism behind the acetaldehyde intoxication is yet to be elucidated.. This means that the cells, even with the abundance of C-source and oxygen in the medium, begin to suffocate, as their ability for anaerobic growth is purposefully deleted. A declining $\mathrm{CO}_{2}$ production was observed in all three

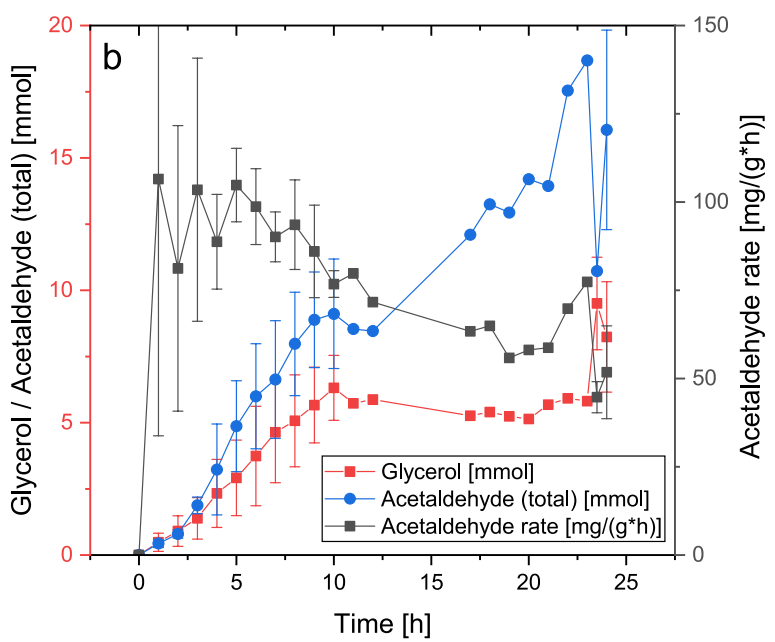

(reactor and traps) produced in the 200-ml setup, and rate of acetaldehyde production cumulated from the beginning of the fermentation as (acetaldehyde ${ }_{t n}-$ acetaldehyde $\left._{t 0}\right) /\left(t_{n}-t_{0}\right) / C D W_{t n} n=3$ for h $0-3,24 ; n=2$ for $4-10,23$ and $n=1$ for $12-23$ ). For all individual figures see $\mathrm{S} 2.7$ 
fermentations, whereby the stationary level of the biomass concentration varied with the inaccuracy in inoculation and the low maximum growth rate in the reactor. This is because, although the acetaldehyde production is primarily dependent on the biomass, the acetaldehyde concentration in the reactor in the first part of the fermentation is also time dependent, as the vessel is saturating. Even though a considerable amount of acetaldehyde is stripped with the gas stream, an accumulation to toxic levels was not prevented in this proof-of-concept setup.

The acetaldehyde in the off-gas stream is captured in a series of water traps. The amount of acetaldehyde produced over the course of the experiment is $12 \mathrm{mmol}$ or $0.55 \mathrm{~g}$ on average (see Fig. 4b). And no traces of other components were found in the GC measurements. As already mentioned, we calculated the capture efficiency to be $75 \%$. Considering the loss of $25 \%$ of product, up to $16 \mathrm{mmol}$ or $0.71 \mathrm{~g}$ of acetaldehyde were produced (for the calculations see S2.6).

Taking a closer look at the dynamics of acetaldehyde production, the specific production rate is depicted in Fig. 4b (for a more detailed calculation see S2.7). The rate stays constant at around $100 \mathrm{mg} / \mathrm{g} / \mathrm{h}$ in the first $6 \mathrm{~h}$ of the fermentation. After that the rate slows down to at least $50 \mathrm{mg} / \mathrm{g} / \mathrm{h}$. Because acetaldehyde derives from the primary metabolism, it is expected to be produced during all stages of the fermentation.

After glycolysis, the pyruvate could either flow towards acetaldehyde production or respiration (TCA-cycle). The distribution of $\mathrm{C}$-atoms between these two options is dependent on the bottleneck respiratory capacity. A higher glycolytic rate and lower respiration capacity should increase the bottleneck and thus shunt more carbon into the production of acetaldehyde. Since it is assumed that acetaldehyde negatively influences the respiratory chain [27], an increased production rate is to be expected.

However, the experimental data shows a decline in the acetaldehyde production rate, of which possible reasons are shortly discussed here. For once, the metabolite could interfere with glucose uptake, glycolysis, or the pyruvate decarboxylases, but studies find only minor implications on the anaerobic $\mathrm{CO}_{2}$ production of $S$. cerevisiae strains stressed with acetaldehyde [38]. The second possibility is a simple end-product inhibition, as acetaldehyde has a low diffusion rate out of the cell and thus can accumulate multiple-fold compared to outside concentration [39]. To access this question, whether the observed concentration of 10 to $15 \mathrm{mmol} / \mathrm{L}$ acetaldehyde in the supernatant is indeed a limit, experiments without gas stripping were performed. The inoculated medium was cultivated in sealed Hungate-tubes with residual air for $24 \mathrm{~h}$. This yielded titers of over $50 \mathrm{mmol} / \mathrm{L}$ acetaldehyde (for more detailed results see S2.8), not supporting the hypothesis of end-product inhibition. This leads to a third explanation: an impaired redox balance.
As seen in Fig. 1, sources of NADH are glycolysis, the pyruvate dehydrogenase, and the TCA cycle, while the major sink is the respiratory chain. As in Crabtree-positive yeasts, glycolysis outruns the respiratory capacity, reduction equivalents usually are additionally regenerated in the acetaldehyde to ethanol conversion [25, 28]. This is possible as ethanol $\left(\gamma_{x}=6.0\right)$ has a higher degree of reduction than glucose $\left(\gamma_{x}=4.0\right)$. Since the genes encoding the enzymes catalysing this reaction are deleted in the present strain, it must use alternative pathways to produce other reduced substances, such as glycerol $\left(\gamma_{x}=4.7\right)$. Here, one $\mathrm{NAD}^{+}$is regenerated at the cost of one pyruvate that cannot be converted into biomass or acetaldehyde. The reason for the probable redox disequilibrium during the fermentation should be attributable to the two main products: biomass $\left(\gamma_{x}=4.17\right)$ and acetaldehyde $\left(\gamma_{x}=5.0\right)$. Thus, the glycerol concentration in the medium increases rapidly in the first $10 \mathrm{~h}$ of the fermentation, where biomass and acetaldehyde are produced (see Fig. 4b). At the point of arrested biomass production, the rate slows down, but glycerol is produced until the end of the fermentation, caused by continuous acetaldehyde production (see Fig. S2.6f. The interesting effects of the redox imbalance should be further investigated, for example, with the determination of intracellular metabolite and cofactor concentrations or metabolic flux analysis.

\section{Conclusion}

The production of the platform chemical acetaldehyde using renewable materials by genetically modified $S$. cerevisiae is possible. We could, at rates between 50 and $100 \mathrm{mg} / \mathrm{g} / \mathrm{h}$ produce on average $0.7 \mathrm{~g}$ acetaldehyde in $200 \mathrm{ml}$ scale, which corresponds to a yield of $38 \%$. In contrast to other publications [16-19], the presented strain does not form ethanol as a side product. We also integrate in situ gas stripping in the fermentation and consecutive absorption of the acetaldehyde. Water was chosen as a suitable solvent. The Henry coefficient for acetaldehyde in water was further determined experimentally, and the obtained value agreed with the predicted value. Based on this, a water trap system was designed, and a near constant capture efficiency of approximately $75 \%$ for a range of possibly produced amounts of acetaldehyde was observed. A balance in the reactor of production and removal was reached at a concentration of 10 to $15 \mathrm{mmol} / \mathrm{L}$ acetaldehyde. It is well known that acetaldehyde acts respiratory toxic, but the exact mechanism of aerobic acetaldehyde intoxication is still elusive. Due to the respiratory toxicity effect of acetaldehyde, a decrease in $\mathrm{CO}_{2}$ production and arrested growth in still excess glucose and oxygenic conditions was observed.

The high glycerol production, even at arrested growth, implies an impaired redox balance. The NADH produced 
during glycolysis cannot be regenerated in the conversion to ethanol, as this activity is absent in the engineered yeast. Regeneration by respiration is hindered when the acetaldehyde threshold in the cells is reached, as the compound is known to inhibit respiration. Glycerol is generally regarded as an unwanted side product. There are numerous approaches to prevent the formation, of which multiple could be applied for future acetaldehyde production studies [40].

In summary, we have performed a new approach of bioacetaldehyde production within $S$. cerevisiae. We successfully used an alcohol dehydrogenase deficient strain in a new lab-scale setup to produce as well as capture acetaldehyde without the co-production of ethanol. Further, we gained new insights on the production dynamics and the challenges to overcome in the future.

Supplementary Information The online version contains supplementary material available at https://doi.org/10.1007/s00449-022-02697-w

\begin{abstract}
Acknowledgements This work is supported by the Deutsche Forschungsgemeinschaft (DFG, German Research Foundation) under Germany's Excellence Strategy - Exzellenzcluster 2186 “The Fuel Science Center" ID: 390919832.
\end{abstract}

Funding Open Access funding enabled and organized by Projekt DEAL.

\section{Declarations}

Conflict of interest The authors have no conflicts of interest to declare that are relevant to this article.

Open Access This article is licensed under a Creative Commons Attribution 4.0 International License, which permits use, sharing, adaptation, distribution and reproduction in any medium or format, as long as you give appropriate credit to the original author(s) and the source, provide a link to the Creative Commons licence, and indicate if changes were made. The images or other third party material in this article are included in the article's Creative Commons licence, unless indicated otherwise in a credit line to the material. If material is not included in the article's Creative Commons licence and your intended use is not permitted by statutory regulation or exceeds the permitted use, you will need to obtain permission directly from the copyright holder. To view a copy of this licence, visit http://creativecommons.org/licenses/by/4.0/.

\section{References}

1. European Commission (2020) Making the EU climate-neutral by 2050. https://ec.europa.eu/commission/presscorner/detail/en/ip_ 20_335

2. Cronshaw M (2021) Petrochemicals. In: Cronshaw M (ed) Energy in perspective, 1st edn. Springer International Publishing, Cham, pp 179-187

3. Mohd Azhar SH, Abdulla R, Jambo SA et al (2017) Yeasts in sustainable bioethanol production: a review. BB Rep 10:52-61. https://doi.org/10.1016/j.bbrep.2017.03.003
4. Perera M, Chinthaka SDM, Wijayarathna CD et al (2021) Reduction of lag in crude oil degradation by Aspergillus when it is in synergy with Bacillus in biofilm mode. Bioprocess Biosyst Eng. https://doi.org/10.1007/s00449-021-02534-6

5. Claassens NJ, Sánchez-Andrea I, Sousa DZ et al (2018) Towards sustainable feedstocks: a guide to electron donors for microbial carbon fixation. Curr Opin Biotech 50:195-205. https://doi.org/ 10.1016/j.copbio.2018.01.019

6. Asenjo JA (ed) (1990) Separation processes in biotechnology. Bioprocess technology, 9th edn. Dekker, New York

7. Eckert M, Fleischmann G, Jira R et al (2006) Acetaldehyde. Ullmann's Encycl Ind Chem. https://doi.org/10.1002/14356007.a01_ 031.pub2

8. Verified Market Research (2021) Global acetaldehyde market size and forecast to 2026. https://www.verifiedmarketresearch.com/ product/acetaldehyde-market/. Accessed 21 Apr 2021

9. Jira R (2009) Acetaldehyde from ethylene-a retrospective on the discovery of the Wacker process. Angew Chem Int Edit 48:9034 9037. https://doi.org/10.1002/anie.200903992

10. Raynes SJ, Taylor RA (2021) Zinc oxide-modified mordenite as an effective catalyst for the dehydrogenation of (bio)ethanol to acetaldehyde. Sustain Energy Fuels. https://doi.org/10.1039/ D1SE00091H

11. Zhang H, Tan H-R, Jaenicke S et al (2020) Highly efficient and robust $\mathrm{Cu}$ catalyst for non-oxidative dehydrogenation of ethanol to acetaldehyde and hydrogen. J Catal 389:19-28. https://doi. org/10.1016/j.jcat.2020.05.018

12. Amrollahi R, Wenderich K, Mul G (2016) Room temperature oxidation of ethanol to acetaldehyde over Pt/WO 3. Adv Mater Interfaces 3:1600266. https://doi.org/10.1002/admi.201600266

13. Pörner Ingenieurgesellschaft mbH (2018) Pörner formaldehyde and derivatives. https://www.poerner.at/fileadmin/user_upload/ downloads/POE/POE_Formaldehyde_Brochure_2018_web.pdf

14. Straathof A (2011) The proportion of downstream costs in fermentative production processes. Comprehensive biotechnology, 2nd edn. Elsevier, Amsterdam, pp 811-814

15. Neuberg C, Reinfurth E (1919) Weitere Untersuchungen über die korrelative Bildung von Acetaldehyd und Glycerin bei der Zuckerspaltung und neue Beiträge zur Theorie der alkoholischen Gärung. Ber dtsch Chem Ges A/B 52:1677-1703. https:// doi.org/10.1002/cber.19190520831

16. Wecker MS, Zall RR (1987) Production of acetaldehyde by Zymomonas mobilis. Appl Environ Microbiol 53:2815-2820. https://doi.org/10.1128/AEM.53.12.2815-2820.1987

17. Zall RR, Wecker M (2021) Microbiological production of acetaldehyde(US4900670A). https://patents.google.com/patent/ US4900670? oq=microbiological+production+of+acetaldehy de. Accessed 03 Sep 2021

18. Tanaka H, Ishikawa H, Osuga K et al (1990) Fermentative ability of Zymomonas mobilis under various oxygen supply conditions in batch culture. J Ferment Bioeng 69:234-239. https:// doi.org/10.1016/0922-338X(90)90219-M

19. Kalnenieks U, Balodite E, Strähler S et al (2019) Improvement of acetaldehyde production in Zymomonas mobilis by engineering of its aerobic metabolism. Front Microbiol 10:2533. https:// doi.org/10.3389/fmicb.2019.02533

20. Balagurunathan B, Tan L, Zhao H (2018) Metabolic engineering of Escherichia coli for acetaldehyde overproduction using pyruvate decarboxylase from Zymomonas mobilis. Enzyme Microb Tech 109:58-65. https://doi.org/10.1016/j.enzmictec.2017.09. 012

21. Bongers RS, Hoefnagel MHN, Kleerebezem M (2005) High-level acetaldehyde production in Lactococcus lactis by metabolic engineering. Appl Environ Microbiol 71:1109-1113. https://doi.org/ 10.1128/AEM.71.2.1109-1113.2005 
22. Brady D, Pratt GC (2007) Volatile organic compound emissions from dry mill fuel ethanol production. J Air Waste Manag Assoc 57:1091-1102. https://doi.org/10.3155/1047-3289.57.9.1091

23. Baerns M (2013) Technische chemie, 2nd edn. Wiley, Hoboken

24. Eliasson J (2010) Design of an plant for manufacturing of acetaldehyde. https://www.semanticscholar.org/paper/Design-of-anPlant-for-Manufacturing-of-Eliasson/4eb877e107e0797438f4 0268369f2510c6321a6d\#references

25. Verduyn C, Zomerdijk TPL, van Dijken JP et al (1984) Continuous measurement of ethanol production by aerobic yeast suspensions with an enzyme electrode. Appl Microbiol Biotechnol 19:181185. https://doi.org/10.1007/BF00256451

26. Halbfeld C (2018) What happens in yeast during the Crabtree effect? : An investigation of $S$. cerevisiae's volatile space, 1 st edn. Wissenschaft Apprimus, Aachen

27. Thielen J, Ciriacy M (1991) Biochemical basis of mitochondrial acetaldehyde dismutation in Saccharomyces cerevisiae. J Bacteriol 173:7012-7017. https://doi.org/10.1128/jb.173.21.7012-7017. 1991

28. Verduyn C, Postma E, Scheffers WA et al (1992) Effect of benzoic acid on metabolic fluxes in yeasts: a continuous-culture study on the regulation of respiration and alcoholic fermentation. Yeast 8:501-517. https://doi.org/10.1002/yea.320080703

29. Benz GT (2008) Piloting bioreactors for agitation scale-up. Chem Eng Prog 104:32-34

30. Scheffczyk JD (2022) Integrated computer-aided design of molecules and processes using COSMO-RS. Dissertation, Günter Mainz

31. Fendinger NJ, Glotfelty DE (1988) A laboratory method for the experimental determination of air-water Henry's law constants for several pesticides. Environ Sci Technol 22:1289-1293. https://doi. org/10.1021/es00176a007

32. Sagebiel JC, Seiber JN, Woodrow JE (1992) Comparison of headspace and gas-stripping methods for determining the Henry's law constant $(\mathrm{H})$ for organic compounds of low to intermediate H. Chemosphere 25:1763-1768. https://doi.org/10.1016/00456535(92)90017-L
33. Coles KF, Popper F (1950) Vapor-liquid equilibria. ethylene oxide - acetaldehyde and ethylene oxide water systems. Ind Eng Chem 42:1434-1438. https://doi.org/10.1021/ie50487a046

34. Yamashita K, Noguchi M, Mizukoshi A et al (2010) Acetaldehyde removal from indoor air through chemical absorption using L-cysteine. Int J Environ Res Public Health 7:3489-3498. https:// doi.org/10.3390/ijerph7093489

35. Eckert F, Klamt A (2002) Fast solvent screening via quantum chemistry: COSMO-RS approach. AIChE J 48:369-385. https:// doi.org/10.1002/aic.690480220

36. Lucas K (2008) Thermodynamik: Die Grundgesetze der Energieund Stoffumwandlungen. Springer, Berlin (Springer-Lehrbuch)

37. Salvadó Z, Arroyo-López FN, Guillamón JM et al (2011) Temperature adaptation markedly determines evolution within the genus Saccharomyces. Appl Environ Microbiol 77:2292-2302. https:// doi.org/10.1128/AEM.01861-10

38. Carlsen HN, Degn H, Lloyd D (1991) Effects of alcohols on the respiration and fermentation of aerated suspensions of baker's yeast. J Gen Microbiol 137:2879-2883. https://doi.org/10.1099/ 00221287-137-12-2879

39. Stanley GA, Pamment NB (1993) Transport and intracellular accumulation of acetaldehyde in Saccharomyces cerevisiae. Biotechnol Bioeng 42:24-29. https://doi.org/10.1002/bit.260420104

40. Naghshbandi MP, Tabatabaei M, Aghbashlo M et al (2019) Progress toward improving ethanol production through decreased glycerol generation in Saccharomyces cerevisiae by metabolic and genetic engineering approaches. Renew Sustain Energy Rev 115:109353. https://doi.org/10.1016/j.rser.2019.109353

Publisher's Note Springer Nature remains neutral with regard to jurisdictional claims in published maps and institutional affiliations. 